\title{
Adaptive control-based voltage regulation of a magnetically coupled multiport dc-dc converter for electrified vehicles applications
}

\author{
Josep M. Olm, Enric Fossas, Victor Repecho, Arnau Dòria-Cerezo and Robert Griñó \\ Institute of Industrial and Control Engineering \\ Universitat Politècnica de Catalunya \\ Barcelona, Spain \\ \{josep.olm,enric.fossas,victor.repecho.del,arnau.doria,roberto.grino $\} @$ upc.edu
}

\begin{abstract}
This paper presents an adaptive control-based strategy to regulate the output voltage of a a magnetically coupled multiport dc-dc converter aimed at automotive applications. The proposed algorithm renders robustness to resistive load uncertainty and to the presence of unknown constant power loads. The theoretical analysis, which uses Lyapunov stability theory, is validated via realistic numerical simulations.
\end{abstract}

Index Terms-Multiport dc-dc converter, adaptive control, automotive applications

\section{INTRODUCTION}

A major increase in the use of electrified systems is seen as a viable option by OEMs (Original Equipment Manufacturers) to face the current main challenges in the automotive sector, namely, $\mathrm{CO}_{2}$ reduction, performance enhancement, and security improvements [1], [2].

However, this entails additional issues on on-board dc microgrids, mainly related to to stability, in particular, to the connection of constant power loads (CPLs), robustness and efficiency.

Aiming at size and weight reduction, this article proposes a dc multi-bus architecture composed by a single storage element and capable of delivering the most commonly used voltages in automotive applications: $400 \mathrm{~V}$ for the power train [3], [4], and $48 \mathrm{~V}$ and $12 \mathrm{~V}$, for auxiliary devices, see Figure 1.

The dc buses are connected by a magnetically coupled multiport dc-dc converter $(400 / 48 / 12 \mathrm{~V} \mathrm{dc})$. This type of dcdc power converters are being used not only for automotive applications [5], but also in electric aircrafts [6], satellites [7], energy management systems [8], and dc grids [9], among others.

So far, the reported techniques on the control of magnetically coupled dc-dc converters are based on a linear approximated model and encompass classical PI controllers [6], [10], PI controllers based on the Generalized State-Space Averaging (GSSA) decomposition [11], decoupling methods [12], [13] and compensators like a two-pole one-zero compensator [14] and a three-pole two-zero compensator [15].

The adaptive control-based strategy presented in this paper considers the inherent nonlinearities of the model and provides

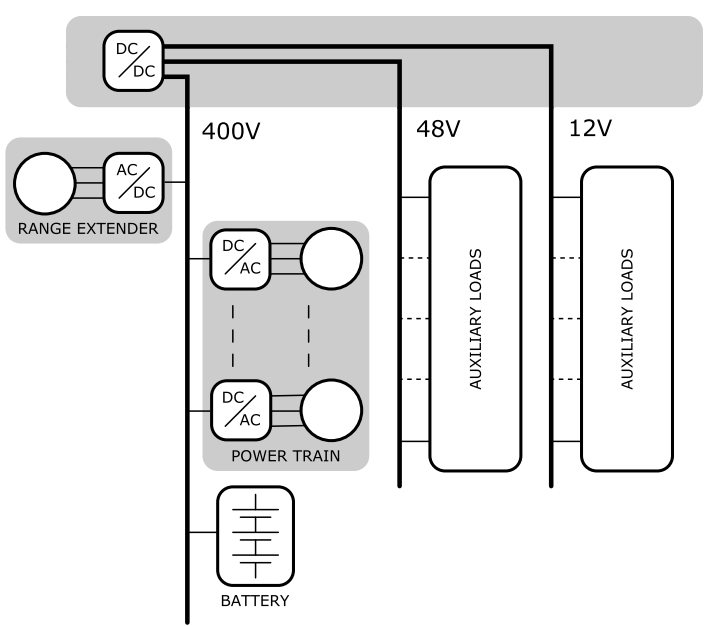

Fig. 1. Scheme of a dc-dc multi-port converter.

energy management among the dc buses of the multiport converter, as well as ac/dc charging functionalities. Essentially, the control action is firstly transformed using a nonlinear change of variables, and an adaptive controller rendering asymptotic voltage regulation and robustness to resistive load disturbances and presence of uncertain CPLs is subsequently proposed. The stability results are derived using LaSalle's invariance principle. Finally, the control transformation is inverted using a linear approximation, which does not entail a significant performance decay. Realistic numerical simulations validate the proposal.

The paper is organized as follows. The mathematical model of the multiport dc-dc converter is presented in Section II. The adaptive controller is proposed in Section III. Simulation results are collected in Section IV and, finally, conclusions and comments on further research lines are drawn in Section V.

\section{Mathematical Model}

A generic magnetically coupled multiport dc-dc converter is shown in Figure 2. It is composed of an $n$-port high frequency transformer with a full-bridge converter and a capacitor in each port side, with the subindex $k \in\{1,2, \ldots, n\}$ indicating the 


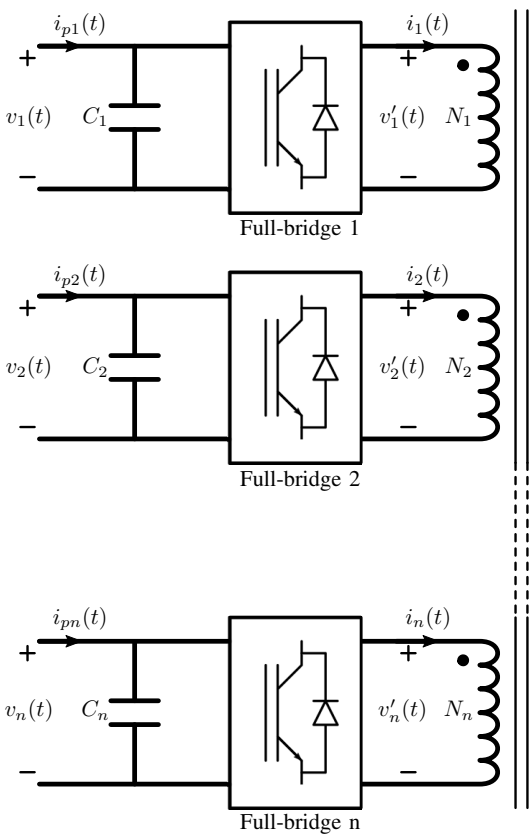

Fig. 2. Multiport dc-dc converter.

port number. DC voltages and currents are denoted by $v_{k}, i_{p k}$, $C_{k}$ stand for the capacities, $v_{k}^{\prime}, i_{k}$ are the high frequency voltages and currents in the transformer, and $N_{k}$ are the number of coils in each winding. Following the behavioural approximation in [16], the dc voltage dynamics answer to

$$
C_{k} \dot{v}_{k}=i_{p k}-\frac{1}{\omega_{1}} \sum_{l=1, l \neq k}^{n} \frac{v_{l}}{\alpha_{k l} L_{k l}^{\prime}} \delta_{k l}\left(1-\frac{\left|\delta_{k l}\right|}{\pi}\right),
$$

where $\omega_{1}=\frac{2 \pi}{T}$ is the fundamental frequency of the periodic voltages and currents in the transformer, $\alpha_{k l}$ is the transformer turn ratio of the $k$-th port with respect to the $l$-th port, $L_{k l}^{\prime}$ is the meshed-transformed linking inductance between port $k$ and any $l$-th port, and $\delta_{k l}=\theta_{l}-\theta_{k}$ is the difference between the phase shifts of the modulated voltages.

Let us consider a three-port dc bus system: a high voltage dc bus, with nominal value $400 \mathrm{~V}$, and two low voltage buses, with nominal values $48 \mathrm{~V}$ and $12 \mathrm{~V}$. The voltage at the $400 \mathrm{~V}$ bus is assumed constant (because of the usual high capacity battery in these buses), while the other buses have several load connections. Setting the phase shift for port 1 as the reference, i.e., $\theta_{1}=0$, the system dynamics answer to

$$
\begin{aligned}
C_{2} \dot{v}_{2}= & -\frac{v_{2}}{R_{2}}-\frac{P_{2}}{v_{2}}+\frac{E_{1}}{\omega_{1} \alpha_{12} L_{12}^{\prime}} \theta_{2}\left(1-\frac{\left|\theta_{2}\right|}{\pi}\right)+ \\
& -v_{3} \frac{1}{\omega_{1} \alpha_{23} L_{23}^{\prime}}\left(\theta_{3}-\theta_{2}\right)\left(1-\frac{\left|\theta_{3}-\theta_{2}\right|}{\pi}\right) \\
C_{3} \dot{v}_{3}= & -\frac{v_{3}}{R_{3}}-\frac{P_{3}}{v_{3}}+\frac{E_{1}}{\omega_{1} \alpha_{13} L_{13}^{\prime}} \theta_{3}\left(1-\frac{\left|\theta_{3}\right|}{\pi}\right)+ \\
& +v_{2} \frac{1}{\omega_{1} \alpha_{32} L_{32}^{\prime}}\left(\theta_{3}-\theta_{2}\right)\left(1-\frac{\left|\theta_{3}-\theta_{2}\right|}{\pi}\right),
\end{aligned}
$$

where $v_{2}, v_{3}$ are the voltages of the $48 \mathrm{~V}$ bus and the $12 \mathrm{~V}$ bus, respectively, while the phase shifts in ports 2 and 3, i.e. $\theta_{2}, \theta_{3}$, are the control variables, with $\theta_{k} \in\left[-\frac{\pi}{2}, \frac{\pi}{2}\right], k \in\{2,3\}$.

\section{AdAPTIVE CONTROL ALGORITHM}

The control goal is to regulate the de voltages $v_{2}, v_{3}$ to the reference values, $v_{2}^{*}=48 \mathrm{~V}$ and $v_{3}^{*}=12 \mathrm{~V}$, in the face of resistive load and CPL variations in the buses, represented by $R_{k}$ and $P_{k}$. The bus voltages are assumed to be measurable.

Let

$$
\begin{aligned}
f_{k}\left(\theta_{k}\right) & =\frac{E_{1}}{\omega_{1} \alpha_{1 k} L_{1 k}^{\prime}} \theta_{k}\left(1-\frac{\left|\theta_{k}\right|}{\pi}\right), \quad k=2,3 \\
g\left(\theta_{2}, \theta_{3}\right) & =\left(\theta_{3}-\theta_{2}\right)\left(1-\frac{\left|\theta_{3}-\theta_{2}\right|}{\pi}\right),
\end{aligned}
$$

and $\lambda_{k}=\left(\omega_{1} \alpha_{k l} L_{k l}^{\prime}\right)^{-1}, k, l=2,3, k \neq l$; let also $G_{k}=$ $R_{k}^{-1}, k=2,3$ denote the conductances in ports 2 and 3 . Then, (2) becomes:

$$
\begin{aligned}
& C_{2} \dot{v}_{2}=-G_{2} v_{2}-\frac{P_{2}}{v_{2}}+f_{2}\left(\theta_{2}\right)-\lambda_{2} v_{3} g\left(\theta_{2}, \theta_{3}\right) \\
& C_{3} \dot{v}_{3}=-G_{3} v_{3}-\frac{P_{3}}{v_{3}}+f_{3}\left(\theta_{3}\right)+\lambda_{3} v_{2} g\left(\theta_{2}, \theta_{3}\right) .
\end{aligned}
$$

Let us now consider the change of control variables

$$
\begin{aligned}
& u_{2}=f_{2}\left(\theta_{2}\right)-\lambda_{2} v_{3}^{*} g\left(\theta_{2}, \theta_{3}\right) \\
& u_{3}=f_{3}\left(\theta_{3}\right)+\lambda_{3} v_{2}^{*} g\left(\theta_{2}, \theta_{3}\right),
\end{aligned}
$$

which, denoting $u=\left(u_{2}, u_{3}\right)^{\top}, \theta=\left(\theta_{2}, \theta_{3}\right)^{\top}$, can be written in compact form as $u=h(\theta)$, with $h:\left[-\frac{\pi}{2}, \frac{\pi}{2}\right]^{2} \longrightarrow \mathbb{R}^{2}$ appropriately defined componentwise using (4a),(4b). Finally, let us assume that $h$ is invertible in a region of interest, and define $H=g \circ h^{-1}$. Then, in the error variables $e_{k}=v_{k}-v_{k}^{*}$, $k=2,3$, (3) boils down to

$$
\begin{aligned}
& C_{2} \dot{e}_{2}=-G_{2}\left(e_{2}+v_{2}^{*}\right)-\frac{P_{2}}{e_{2}+v_{2}^{*}}-\lambda_{2} e_{3} H(u)+u_{2} \\
& C_{3} \dot{e}_{3}=-G_{3}\left(e_{3}+v_{3}^{*}\right)-\frac{P_{3}}{e_{3}+v_{3}^{*}}+\lambda_{3} e_{2} H(u)+u_{3} .
\end{aligned}
$$

Proposition 1. Let $G_{k}, P_{k} \geq 0, k=2,3$, in (5) be constant, unknown conductances and CPLs, respectively, and let $\hat{G}_{k}, \hat{P}_{k}$, denote their estimated values. Then, the adaptive control law

$$
\begin{aligned}
u_{k} & =\hat{G}_{k}\left(e_{k}+v_{k}^{*}\right)+\frac{\hat{P}_{k}}{e_{k}+v_{k}^{*}}-\gamma_{k} e_{k}, \\
\dot{\hat{G}}_{k} & =-\frac{\mu_{k}}{\lambda_{k}} e_{k}\left(e_{k}+v_{k}^{*}\right), \\
\dot{\hat{P}}_{k} & =-\frac{\nu_{k} e_{k}}{\lambda_{k}\left(e_{k}+v_{k}^{*}\right)},
\end{aligned}
$$

with $\gamma_{k}, \mu_{k}, \nu_{k} \in \mathbb{R}^{+}, k=2,3$, makes the closed-loop system (5),(6) locally stable and $e_{k} \rightarrow 0, k=2,3$, when $t \rightarrow+\infty$.

Proof. Let $\Delta G_{k}=G_{k}-\hat{G}_{k}, \Delta P_{k}=P_{k}-\hat{P}_{k}, k=2,3$, denote the estimation errors for conductances and CPLs. As 
$G_{k}, P_{k}$ are assumed constant, it is immediate that, for $k=2,3$, $\dot{\Delta} G_{k}=-\dot{\hat{G}}_{k}, \dot{\Delta} P_{k}=-\dot{\hat{P}}_{k}$. Therefore, (5),(6) reads as:

$$
\begin{aligned}
& C_{2} \dot{e}_{2}=-\Delta G_{2}\left(e_{2}+v_{2}^{*}\right)-\frac{\Delta P_{2}}{e_{2}+v_{2}^{*}}-\lambda_{2} e_{3} H(u)-\gamma_{2} e_{2} \\
& C_{3} \dot{e}_{3}=-\Delta G_{3}\left(e_{3}+v_{3}^{*}\right)-\frac{\Delta P_{3}}{e_{3}+v_{3}^{*}}+\lambda_{3} e_{2} H(u)-\gamma_{3} e_{3} \\
& \dot{\Delta} G_{k}=\frac{\mu_{k}}{\lambda_{k}} e_{k}\left(e_{k}+v_{k}^{*}\right), \quad k=2,3 \\
& \dot{\Delta} P_{k}=\frac{\nu_{k} e_{k}}{\lambda_{k}\left(e_{k}+v_{k}^{*}\right)}, \quad k=2,3
\end{aligned}
$$

Notice that the auxiliary function

$$
V=\frac{1}{2} \sum_{k=2}^{3}\left(\frac{C_{k}}{\lambda_{k}} e_{k}^{2}+\frac{1}{\mu_{k}} \Delta G_{k}^{2}+\frac{1}{\nu_{k}} \Delta P_{k}^{2}\right)
$$

is positive definite, while $\dot{V}$ is negative semidefinite:

$$
\dot{V}=-\frac{\gamma_{2}}{\lambda_{2}} e_{2}^{2}-\frac{\gamma_{3}}{\lambda_{3}} e_{3}^{2} \leq 0 .
$$

Moreover, the subset of $\mathbb{R}^{6}$ where $\dot{V}=0$ is

$$
\Omega_{R}:=\left\{\left(0,0, \Delta G_{2}, \Delta G_{3}, \Delta P_{2}, \Delta P_{3}\right), \Delta G_{k}, \Delta P_{k} \in \mathbb{R}\right\},
$$

while the largest invariant set within $\Omega_{R}$ is

$$
\Omega:=\left\{\left(0,0, \Delta G_{2}^{*}, \Delta G_{3}^{*}, \Delta P_{2}^{*}, \Delta P_{3}^{*}\right), \Delta P_{k}^{*}=-\Delta G_{k}^{*} v_{k}^{* 2}\right\} .
$$

Then, La Salle's invariance principle ensures that, locally, the trajectories of (7) tend to $\Omega$ for $t \rightarrow+\infty$.

Remark 1. Notice that, although the control law (6) yields asymptotic voltage regulation, the fact that the equilibrium values $\Delta G_{k}^{*}, \Delta P_{k}^{*}, k=2,3$, are not guaranteed to be zero does not allow to identify the real values of conductances and CPLs.

The control law transformation (4) involves absolute value terms. In order to carry out the inversion, i.e. to obtain $\theta=$ $H(u)$, it is important to realize that the $\mathbb{R}^{3}$ surfaces defined in (4) reveal a linear dependence in the region of interest, namely, $u_{k} \in[0,50] \mathrm{A}, k=2,3$, as shown in Figure 3 .

Therefore, the inversion will be performed from the linear approximation of the change of variables (4), namely,

$$
\begin{aligned}
& u_{2} \approx \beta_{2} \theta_{2}-\lambda_{2} v_{3}^{*}\left(\theta_{3}-\theta_{2}\right) \\
& u_{3} \approx \beta_{3} \theta_{3}+\lambda_{3} v_{2}^{*}\left(\theta_{3}-\theta_{2}\right),
\end{aligned}
$$

with $\beta_{k}=E_{1}\left(\omega_{1} \alpha_{1 k} L_{1 k}^{\prime}\right)^{-1}, k=2,3$. Then:

$$
\begin{aligned}
\theta_{2} & \approx \frac{\left(\lambda_{3} v_{2}^{*}+\beta_{3}\right) u_{2}+\lambda_{2} v_{3}^{*} u_{3}}{\lambda_{3} \beta_{2} v_{2}^{*}+\lambda_{2} \beta_{3} v_{3}^{*}+\beta_{2} \beta_{3}} \\
\theta_{3} & \approx \frac{\lambda_{3} v_{2}^{*} u_{2}+\left(\lambda_{2} v_{3}^{*}+\beta_{2}\right) u_{3}}{\lambda_{3} \beta_{2} v_{2}^{*}+\lambda_{2} \beta_{3} v_{3}^{*}+\beta_{2} \beta_{3}},
\end{aligned}
$$

where the control actions $u_{k}$ are defined in (6).
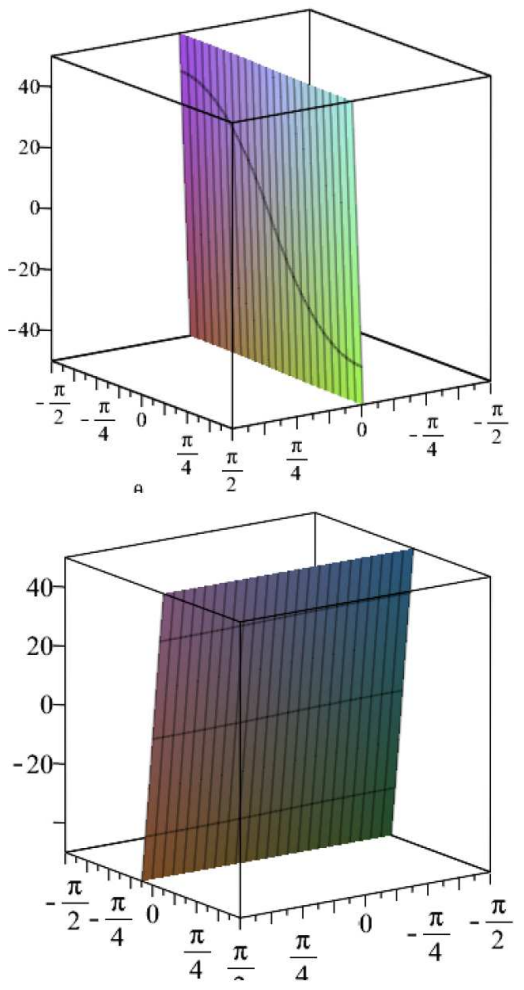

Fig. 3. $u=H(\theta)$. Top: $u_{2}$; bottom: $u_{3}$.

\section{Simulation RESUlts}

The simulations have been carried out with MatlabSimulink, and the DC converter is modelled with SimScape. The solver uses a $10^{-8}$ fixed step size Runge-Kutta ode4 integration algorithm. The converter model includes switching and conduction losses in the power switches, resistive losses in the transformer windings, and reactive components. The branches of the full-bridge converters incorporate a $1 \mu$ s dead time to avoid bus voltage short circuits. First order filters with unitary gain and $2 \mathrm{~ms}$ time constant are placed in the feedback loops. System and control parameters are in Table I.

TABLE I

PARAMETERS FOR SYSTEM (2)

\begin{tabular}{||c|c||c|c||}
\hline$E_{1}$ & $400 \mathrm{~V}$ & $\omega_{1}$ & $2 \pi \cdot 40.0 \cdot 10^{3} \mathrm{rad} / \mathrm{s}$ \\
\hline$C_{2}$ & $200 \mu \mathrm{F}$ & $C_{3}$ & $600 \mu \mathrm{F}$ \\
\hline$\alpha_{12}$ & 0.125 & $\alpha_{13}$ & 0.033 \\
\hline$\alpha_{23}$ & 0.267 & $\alpha_{32}$ & 3.75 \\
\hline$L_{12}^{\prime}$ & $1.27 \mu \mathrm{H}$ & $L_{13}^{\prime}$ & $0.53 \mu \mathrm{H}$ \\
\hline$L_{23}^{\prime}$ & $0.58 \mu \mathrm{H}$ & $L_{32}^{\prime}$ & $34.6 \mu \mathrm{H}$ \\
\hline$\gamma_{2}$ & 4800 & $\gamma_{3}$ & $4.8 \cdot 10^{4}$ \\
\hline$\mu_{2}$ & 51327 & $\mu_{3}$ & 61.327 \\
\hline$\nu_{2}$ & $1.283 \cdot 10^{9}$ & $\nu_{3}$ & $1.5332 \cdot 10^{8}$ \\
\hline
\end{tabular}

Piecewise constant profiles for the resistive loads have been used:

$$
\begin{aligned}
& R_{2}= \begin{cases}5 \Omega & t \in[0,0.03) \cup[0.05,0.1) s \\
1 \Omega & t \in[0.03,0.05) s\end{cases} \\
& R_{3}= \begin{cases}10 \Omega & t \in[0,0.04) \cup[0.06,0.1) s \\
3 \Omega & t \in[0.04,0.06) s .\end{cases}
\end{aligned}
$$



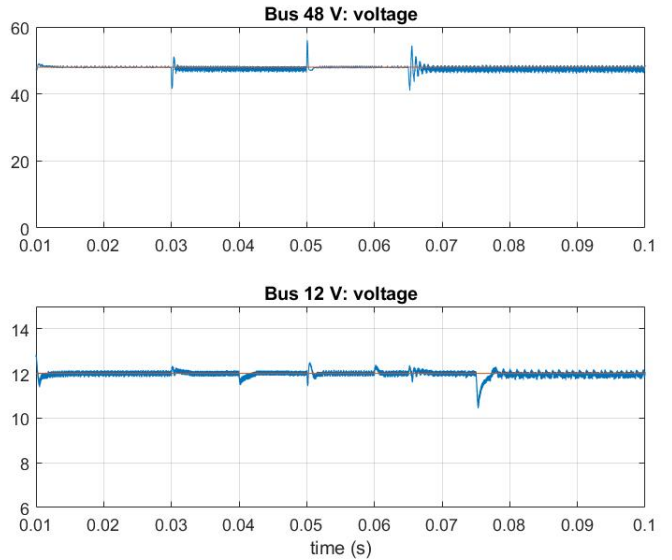

Fig. 4. Bus voltage (blue) and voltage reference (red). Top: $48 \mathrm{~V}$ bus; bottom: $12 \mathrm{~V}$ bus.
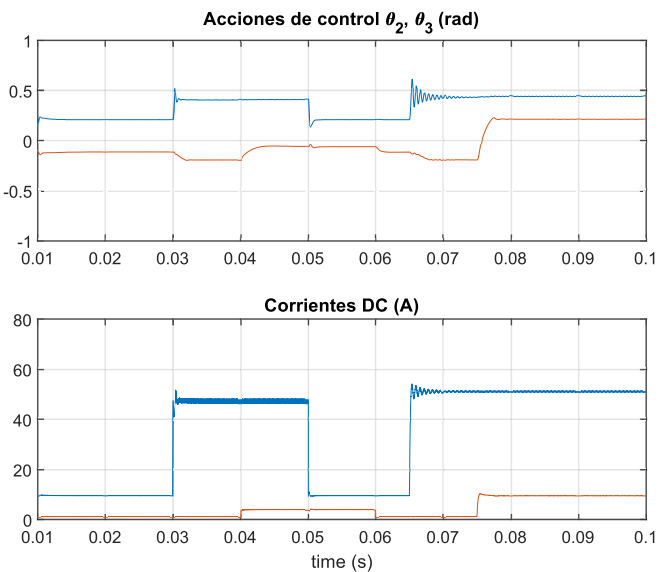

Fig. 5. Top: control actions $\theta_{2}$ (blue), $\theta_{3}$ (red) in radiants; bottom: output currents $-i_{p 2}$ (blue), $-i_{p 3}$ (red) in Ampères.

In turn, piecewise constant CPL profiles connected via first order filters with time constant $0.1 \mathrm{~ms}$ have also been used:

$$
\begin{aligned}
& P_{2}= \begin{cases}0 & t \in[0,0.065) \mathrm{s} \\
2 \mathrm{~kW} & t \in[0.065,0.1) \mathrm{s}\end{cases} \\
& P_{3}= \begin{cases}0 & t \in[0,0.075) \mathrm{s} \\
100 \mathrm{~W} & t \in[0.075,0.1) \mathrm{s}\end{cases}
\end{aligned}
$$

The stability and robustness features of the control design are shown in Figure 4, which portrays the bus voltages tracking their corresponding references. The top part of Figure 5 shows the control action variables reacting in a bounded way to cancel out the effect of the sudden load and CPL changes in the bus voltages. The output currents, $-i_{p 2},-i_{p 3}$ (recall Figure 2), are displayed in the bottom part. The estimated values of the conductances and CPLs are depicted in Figure 6: according to Proposition 1 and Remark 1, all the signals stabilize but not at the real values. Moreover, the flux density at the core of the transformer depicted in Figure 7 guarantees that there are no saturation issues.
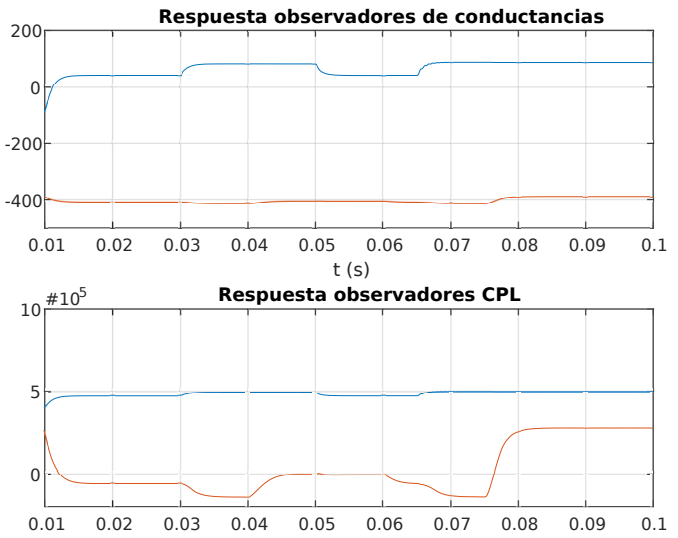

Fig. 6. Observers. Top: estimated conductances $\hat{G}_{2}$ (blue), $\hat{G}_{3}$ (red); bottom: estimated CPLs $\hat{P}_{2}$ (blue), $\hat{P}_{3}$ (red).
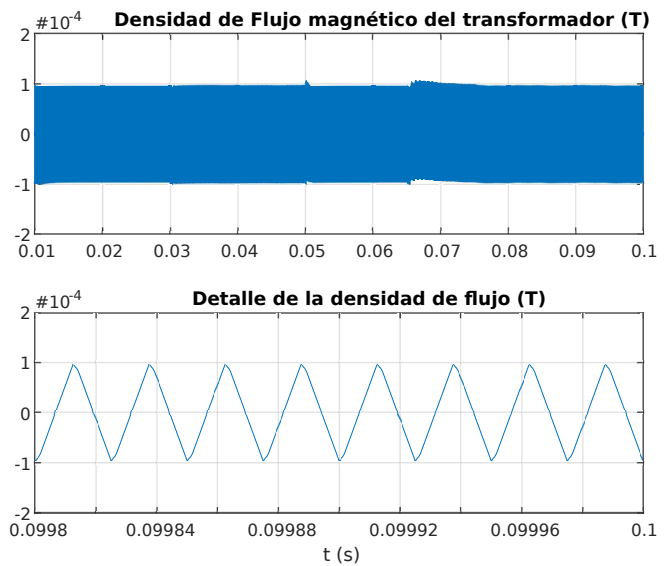

Fig. 7. Top: flux density at the core of the transformer; bottom: detail.

Finally, it turns out that adding a $5 \mu H$ inductance in series with the primary winding with no update of the control parameters, produces essentially the same outcome. This result further illustrates the controller robustness. For the sake of brevity, the plots corresponding to this last case are omitted.

\section{Conclusions}

An adaptive controller was designed for a magnetically coupled dc-dc converter providing asymptotic voltage regulation and robustness in the face of resistive load uncertainty and presence of CPLs. A key role in the control design was played by a nonlinear control transformation that was subsequently inverted using a linear approximation with no significant performance loss. Realistic numerical simulations confirmed the theoretical analysis.

\section{ACKNOWLEDGMENT}

The work was partially supported by the Government of Spain through the Agencia Estatal de Investigación Project DPI2017-85404-P and by the Generalitat de Catalunya through the Project 2017 SGR 872. 


\section{REFERENCES}

[1] "Use of new energies in road transport," European Green Vehicles Initiative. PPP, Tech. Rep., 2013.

[2] "Hybrid and electric vehicles - the electric drive commutes," International Energy Agency, Tech. Rep., 2016.

[3] C. Chan, "The state of the art of electric, hybrid, and fuel cell vehicles," Proc. of the IEEE, vol. 95, no. 4, pp. 704-718, 2007.

[4] O. Coppin, "From $12+12 \mathrm{~V}$ to $48 \mathrm{~V}$ : a new road map for hybridization," in Proc. Engine Expo 2016, 2016.

[5] B. Farhangi and H. Toliyat, "Modeling and analyzing multiport isolation transformer capacitive components for onboard vehicular power conditioners," IEEE Trans. on Industrial Electronics, vol. 62, no. 5, pp. 3134-3142, 2015.

[6] B. Karanayil, M. Ciobotaru, and V. Agelidis, "Power flow management of isolated multiport converter for more electric aircraft," IEEE Trans. on Power Electronics, vol. 32, no. 7, pp. 5850-5861, 2017.

[7] Z. Qian, "Modeling and design of multi-port dc/dc converters," Ph.D. dissertation, University of Central Florida, 2010.

[8] H. Tao, J. Duarte, and M. Hendrix, "Three-port triple-half-bridge bidirectional converter with zero-voltage switching," IEEE Trans. on Power Electronics, vol. 23, no. 2, pp. 782-792, 2008.

[9] H. Matsuo, W. Lin, F. Kurokawa, T. Shigemizu, and N. Watanabe, "Characteristics of the multiple-input DC-DC converter," IEEE Trans. on Industrial Electronics, vol. 51, no. 3, pp. 625 - 631, 2004.

[10] V. Jakka, A. Shukla, and G. Demetriades, "Dual-transformer-based asymmetrical triple-port active bridge (DT-ATAB) isolated DC-DC converter," IEEE Trans. on Industrial Electronics, vol. 64, no. 6, pp. 4549-4560, 2017.

[11] Z. Li, Y. Wang, L. Shi, J. Huang, Y. Cui, and W. Lei, "Generalized averaging modeling and control strategy for three-phase dual-activebridge DC-DC converters with three control variables," in Proc. IEEE Applied Power Electronics Conference and Exposition, 2017.

[12] C. Zhao, S. Round, and J. Kolar, "An isolated three-port bidirectional DC-DC converter with decoupled power flow management," IEEE Trans. on Power Electronics, vol. 23, no. 5, pp. 2443-2453, 2008.

[13] K. Xiangli, S. Li, and K. Smedley, "Decoupled pwm plus phase-shift control for a dual-half-bridge bidirectional dc-dc converter," IEEE Trans. on Power Electronics, vol. 33, no. 8, p. 7203-7213, 2018.

[14] B. Choi, W. Lim, S. Choi, and J. Sun, "Comparative performance evaluation of current-mode control schemes adapted to asymmetrically driven bridge-type pulsewidth modulated dc-to-dc converters," IEEE Trans. on Industrial Electronics, vol. 55, no. 5, p. 2033-2042, 2008.

[15] Z. Qian, O. Abdel-Rahman, H. Al-Atrash, and I. Batarseh, "Modeling and control of three-port DC/DC converter interface for satellite applications," IEEE Trans. on Power Electronics, vol. 25, no. 3, pp. 637-649, 2010.

[16] R. Griñó and A. Dòria-Cerezo, "Modelling and simulation of a magnetically coupled multiport dc-dc converter," in Proc. 24th Int. Conf. on Emerging Technologies and Factory Automation, 2019. 